\title{
Tuning the Photocatalytic Activity of Tin Oxide through Mechanical Surface Activation
}

\author{
Fernando B. F. Silva, ${ }^{a, b}$ Gelson T. S. T. da Silva, ${ }^{\circledR a}$ Juliana A. Torres ${ }^{a}$ and \\ Caue Ribeiro ${ }^{\circledR *, a, b}$
}

${ }^{a}$ Laboratório Nacional de Nanotecnologia para o Agronegócio (LNNA), Embrapa Instrumentação, 13561-206 São Carlos-SP, Brazil

${ }^{b}$ Departamento de Química, Universidade Federal de São Carlos, 13565-905 São Carlos-SP, Brazil

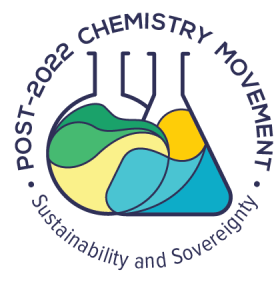

Tin oxide $\left(\mathrm{SnO}_{2}\right)$ nanoparticles were synthesized by the co-precipitation method and mechanically modified by high-energy ball milling. The experimental results demonstrate that the collision with zirconia balls produces slight changes in the crystalline, electronic, morphological, and surface properties of $\mathrm{SnO}_{2}$, which lead to an increase in the redox potential of the energy level and the formation of the hydroxyl group on the $\mathrm{SnO}_{2}$ surface. Moreover, these changes are intensified over the milling up to $90 \mathrm{~min}$, directly affecting the photocatalytic performance, which was monitored by the rate of rhodamine $\mathrm{B}(\mathrm{RhB})$ degradation driven by ultraviolet (UV) irradiation. As a result, all ground samples showed better photocatalytic activity than pristine $\mathrm{SnO}_{2}$ (Sn-cop). The maximum degradation of rhodamine $\mathrm{B}$ was ca. $75 \%$, achieved with 90 min-milled $\mathrm{SnO}_{2}$ nanoparticles (Sn-M90), compared to the Sn-cop sample induced a 1.67 times higher degradation rate. The reaction mechanism suggests that its better photocatalytic activity may be associated with the higher increased redox potential of the valence and conduction bands and the formation of hydroxyl active sites on the catalyst surface principal oxidizing agent generated. Therefore, we conclude that the ball milling process is an efficient way to induce stable activation of oxide metal for photocatalytic applications.

Keywords: $\mathrm{SnO}_{2}$ nanoparticles, mechanical activation, photocatalysis, bandgap dependence, hydroxyl groups

\section{Introduction}

Metal oxide nanoparticles have been widely investigated in photocatalytic processes, playing a crucial role in mitigating the environmental problems generated by greenhouse gases $(\mathrm{GHG})$ emissions and the release of emerging pollutants. ${ }^{1,2}$ Until now, the most studied photocatalyst is titanium dioxide $\left(\mathrm{TiO}_{2}\right)$ because it has favorable optical, electronic, and mechanical properties for this process. ${ }^{3,4}$ Structurally, tin dioxide $\left(\mathrm{SnO}_{2}\right)$ is very similar: a semiconductor with a bandgap of ca. $3.6 \mathrm{eV}$, widely applied as gas sensors and batteries due to its stability, conductivity, and transparency. ${ }^{5}$ Although it has a high oxidation capacity in the valence band, its potential as photocatalyst is somewhat unclear since the $\mathrm{SnO}_{2}$ conduction band energy cannot reduce $\mathrm{H}^{+}$to $\mathrm{H}_{2}$ either

*e-mail: caue.ribeiro@embrapa.br

Editor handled this article: Fernando C. Giacomelli (Associate)
$\mathrm{O}_{2}$ to $\mathrm{O}_{2}{ }^{--}$. It dramatically limits the applicability in the water medium, decreasing the photogenerated electron/ hole charges' lifetime and thus depleting the catalyst performance. $^{6-8}$

It is essential to understand the role of the other properties directly involved in this process to overcome this drawback and adequately take advantage of the preferred characteristics of this material, such as the specific surface area, ${ }^{9}$ crystallinity, ${ }^{10}$ particle size,${ }^{11}$ specific phases, ${ }^{12}$ and the surface chemical composition. ${ }^{13}$ The catalytic properties of nanoparticles are controlled during the synthesis of the materials or altered after preparation from chemical or physical treatments, such as mechanical activation with a ball mill. ${ }^{14,15}$ This strategy provides elastic and inelastic deformations in solids via high-energy collisions between metallic or ceramic micro balls, forming linear defects, such as plane displacement, ionic and atomic vacancies, and interstitial ions. ${ }^{16,17}$ All these changes amplify the binding energies and properties of the intermediate surface species and potentially change the catalytic activity, reactivity, and 
selectivity. ${ }^{18,19}$ For instance, Molchanov et al. ${ }^{20}$ reported that the mechanical treatment of zinc oxide $(\mathrm{ZnO})$ in a planetary mill significantly improved the efficiency of the catalysts to convert carbon monoxide $(\mathrm{CO})$ into carbon dioxide $\left(\mathrm{CO}_{2}\right)$ under UV irradiation through the generation of active sites arising from the formation of defects on the material surface.

Therefore, herein we studied the photoactivity of $\mathrm{SnO}_{2}$ nanoparticles modified by a dry mechanical activation for different times and associated the photocatalytic performance for the degradation of organic molecules under UV irradiation.

\section{Experimental}

\section{Chemicals}

Tin(II) chloride dihydrate $\left(\mathrm{SnCl}_{2} \cdot 2 \mathrm{H}_{2} \mathrm{O}\right)(98 \%)$ and rhodamine $\mathrm{B}(\mathrm{RhB},>95 \%)$ were purchased from Sigma-Aldrich Chemical Company (St. Louis, United States), anhydrous ethanol from Vetec (Duque de Caxias-RJ, Brazil). The deionized water (resistivity below $18.2 \mathrm{M} \Omega \mathrm{cm}$ ) was obtained from a Milli-Q water purification system (Millipore, Bedford, MA, USA).

\section{Synthesis and characterization of the $\mathrm{SnO}_{2}$ nanoparticles}

The synthesis of $\mathrm{SnO}_{2}$ was performed by the typical precipitation method described by Ribeiro et al. ${ }^{21}$ In brief, an ethanolic solution of $\mathrm{SnCl}_{2} \cdot 2 \mathrm{H}_{2} \mathrm{O}(25 \mathrm{mM})$ was prepared, and then distilled water was added drop by drop $(4.5: 1 \mathrm{v} / \mathrm{v}$ (ethanol/water)) under constant stirring. After that, the chloride ion was removed by dialysis, and the colloid dried overnight at $50{ }^{\circ} \mathrm{C}$. For $\mathrm{SnO}_{2}$ surface mechanical activation, $500 \mathrm{mg}$ of prepared oxide nanoparticles and $1.84 \mathrm{~g}$ of zirconia balls (diameter 1-2 $\mathrm{mm}$ ) were loaded into microtubes $(2 \mathrm{~mL})$ and placed into a stainless-steel jar, support of the "nano-grinder" CryoMill (Retsch, Germany). The grinding process was performed at $25^{\circ} \mathrm{C}$ under a vibration frequency of $25 \mathrm{~Hz}$ at different times. The sample without mechanical treatment was named $\mathrm{Sn}$-cop, and the treated samples were called $\mathrm{Sn}-\mathrm{Mx}$, where $\mathrm{x}$ is related to the time used for the treatment time (10, 30, 90, and $150 \mathrm{~min})$, i.e., Sn-M10, Sn-M30, Sn-M90, Sn-M150. The material mass, ball mass, volume, and vibration frequency were kept constant in all grinding procedures. The samples were characterized by X-ray diffraction (XRD) equipment (Shimadzu XRD 6000, Kyoto, Japan). The $\mathrm{X}$-ray source used was $\mathrm{Cu} \mathrm{K} \alpha$ radiation $(\lambda=0.15406 \mathrm{~nm})$. The diffractograms were obtained under a continuous scanning routine, with a $2^{\circ} \mathrm{min}^{-1}$ rate in the $2 \theta$ range from 10 to $70^{\circ}$. Scanning electron microscopy with an electron emission gun (SEM-FEG, field emission gun) was used to observe the morphology of the synthesized materials (JEOL JSM 6701F, Tokyo, Japan). High-resolution transmission electron microscopy (HRTEM, Tecnai G2 F20, FEI, Oregon, United States) was applied to observe the constituent particles of the xerogel. The textural properties of the synthesized samples were performed in ASAP 2020 (Micromeritics Co., USA) equipment. Previously, samples were treated (degassed) by heating at $100{ }^{\circ} \mathrm{C}$ under vacuum until reaching a degassing pressure lower than $10 \mu \mathrm{mHg}$. The samples' specific surface area (SSA) was obtained from the $\mathrm{N}_{2}$ adsorption isotherms in the materials, applying the BET modeling (Brunauer-Emmett-Teller), which considers the adsorption of $\mathrm{N}_{2}$ in multilayers. The sample was suspended in ethanol and dripped onto a copper wire with a diameter of approximately $2 \mathrm{~mm}$, covered with a carbon film known as formvar. Near-infrared spectroscopy (NIR) was used to estimate the covering profile of $\mathrm{OH}$ groups on the surface of the samples through the qualitative determination of the adsorption of water molecules using the PerkinElmer Spectrum 100 equipment (Waltham, MA, United States). The band gaps of the samples synthesized were determined by the Tauc method from diffuse reflectance (DRS) spectra (Shimadzu 2600, Kyoto, Japan), using pressed pellets at room temperature. The photocatalytic performance of the synthesized materials was measured with a Shimadzu UV-1601PC spectrometer (Kyoto, Japan) through the decolorization of rhodamine B $(554 \mathrm{~nm})$.

The photodegradation tests were performed using a cationic dye as a model organic molecule to evaluate the photocatalytic activity of the materials. The assays were performed by dispersing the $\mathrm{SnO}_{2}$ particles in $\mathrm{RhB}$ (5 ppm) solution to form a suspension with a concentration of $200 \mathrm{mg} \mathrm{L}^{-1}$ ( $5 \mathrm{mg}$ of photocatalyst in $25 \mathrm{~mL}$ of $\mathrm{RhB}$ solution). The suspensions were conditioned in a photoreactor coupled to a thermostatic bath at $18{ }^{\circ} \mathrm{C}$. However, before starting the photocatalytic experiments, the suspensions were kept in the dark for $12 \mathrm{~h}$ to establish rhodamine B molecules' adsorption/desorption equilibrium on the active sites located on the photocatalytic surface. Then, the suspensions were irradiated by six TUV-C (Philips) $15 \mathrm{~W}$ lamps with a maximum emission of $254 \mathrm{~nm} .{ }^{22}$ The materials were positioned symmetrically in the reactor, in positions defined by measurements with a radiometer. Finally, the samples were irradiated uniformly to compare the different samples applied in the same cycle.

\section{Results and Discussion}

XRD patterns of pristine and milled $\mathrm{SnO}_{2}$ powders are shown in Figure 1. Broad peaks at $2 \theta$ of $27^{\circ}, 34^{\circ}, 52^{\circ}$, and $65^{\circ}$ correspond to the (110), (101), (211), and (301) planes, 
respectively, suggesting the formation of the tetragonal (cassiterite) phase (JCPDS No. 41-1445). In addition, the enlarged shape and weak intensity of the diffraction peaks indicate the synthesized samples have small crystal size even after the ball mill treatment at different times (10, $30,90$, and $150 \mathrm{~min}){ }^{23}$ At all mechanical friction times, the X-ray diffraction peaks associated with the (211) plane showed a lower value than the $\mathrm{SnO}_{2}$ (Sn-cop) sample, but no significant variations between milling times. This shift indicates that the structural distortion promoted by the collisions between zirconia balls and $\mathrm{SnO}_{2}$ leads to stressenergy on the particle resulting in strain and expansion of the $\mathrm{SnO}_{2}$ crystal lattice. ${ }^{24-26}$ The calculated crystallite sizes using the Scherrer equation (see Table 1) demonstrate

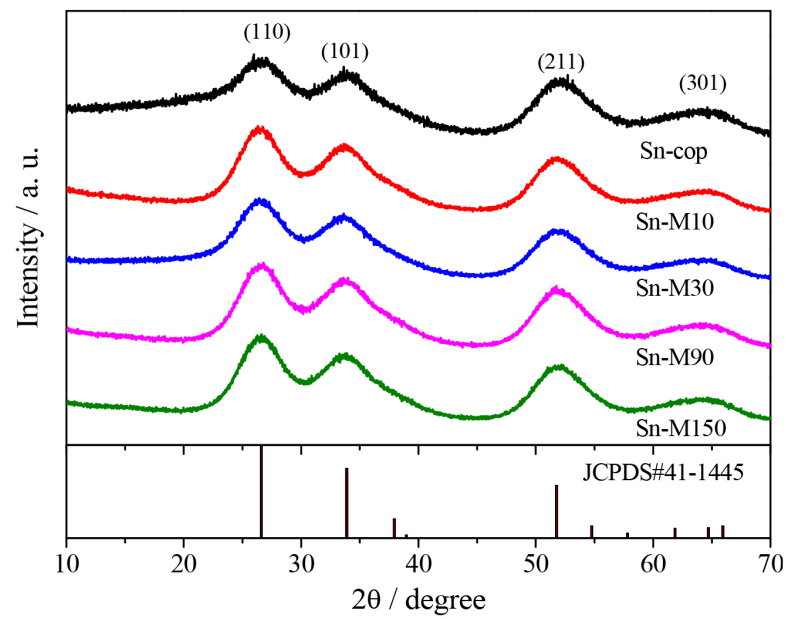

Figure 1. XRD patterns of the tetragonal phase (cassiterite) of as-prepared $\mathrm{SnO}_{2}$ nanoparticles processed by milling for different times, from 0 (Sn-cop) to $150 \mathrm{~min}$ (M150).
Table 1. Crystallite size (D-Scherrer) calculated by Scherrer equation and the specific surface area (SSA) of the synthesized $\mathrm{SnO}_{2}$ samples

\begin{tabular}{lccc}
\hline Material & 20/degree & D-Scherrer $/ \mathrm{nm}$ & $\begin{array}{c}\text { BET surface } \\
\text { area } /\left(\mathrm{m}^{2} \mathrm{~g}^{-1}\right)\end{array}$ \\
\hline Sn-cop & 52.23 & 4.00 & 188.03 \\
Sn-M10 & 52.07 & 3.91 & 165.02 \\
Sn-M30 & 52.06 & 3.94 & 151.23 \\
Sn-M90 & 52.13 & 3.58 & 127.49 \\
Sn-M150 & 52.14 & 3.85 & 131.19 \\
\hline
\end{tabular}

BET: Brunauer-Emmett-Teller.

that friction and plastic deformation from the high-energy milling process produces small crystallite sizes. ${ }^{27}$

Although the milling process does not exhibit pronounced short-term order changes, as phase transition, ${ }^{28,29}$ it is possible to observe that the friction strategy can promote changes in the bulk material, as demonstrated in the representative images (Figure 2). Sn-cop morphologies (Figure 2a) are composed of faceted xerogel blocks, i.e., agglomerates of $4 \mathrm{~nm}$-spherical nanoparticles (Figure 2f). ${ }^{21,23}$ The continuous mechanical friction from 10 to $150 \mathrm{~min}$ produces different deformations in the larger agglomerates, causing higher stress between the smaller particles and breaking the agglomerates, i.e., the collision energy is higher than the attraction energy between the particles. ${ }^{30}$ Thus, the larger particles are broken up with increasing grinding time, and the smaller ones distribute themselves homogeneously. Indeed, secondary agglomerates can be seen just in 10 min.

Table 1 shows the dependence of specific surface area (SSA) of $\mathrm{SnO}_{2}$ on mechanical treatment. The textural
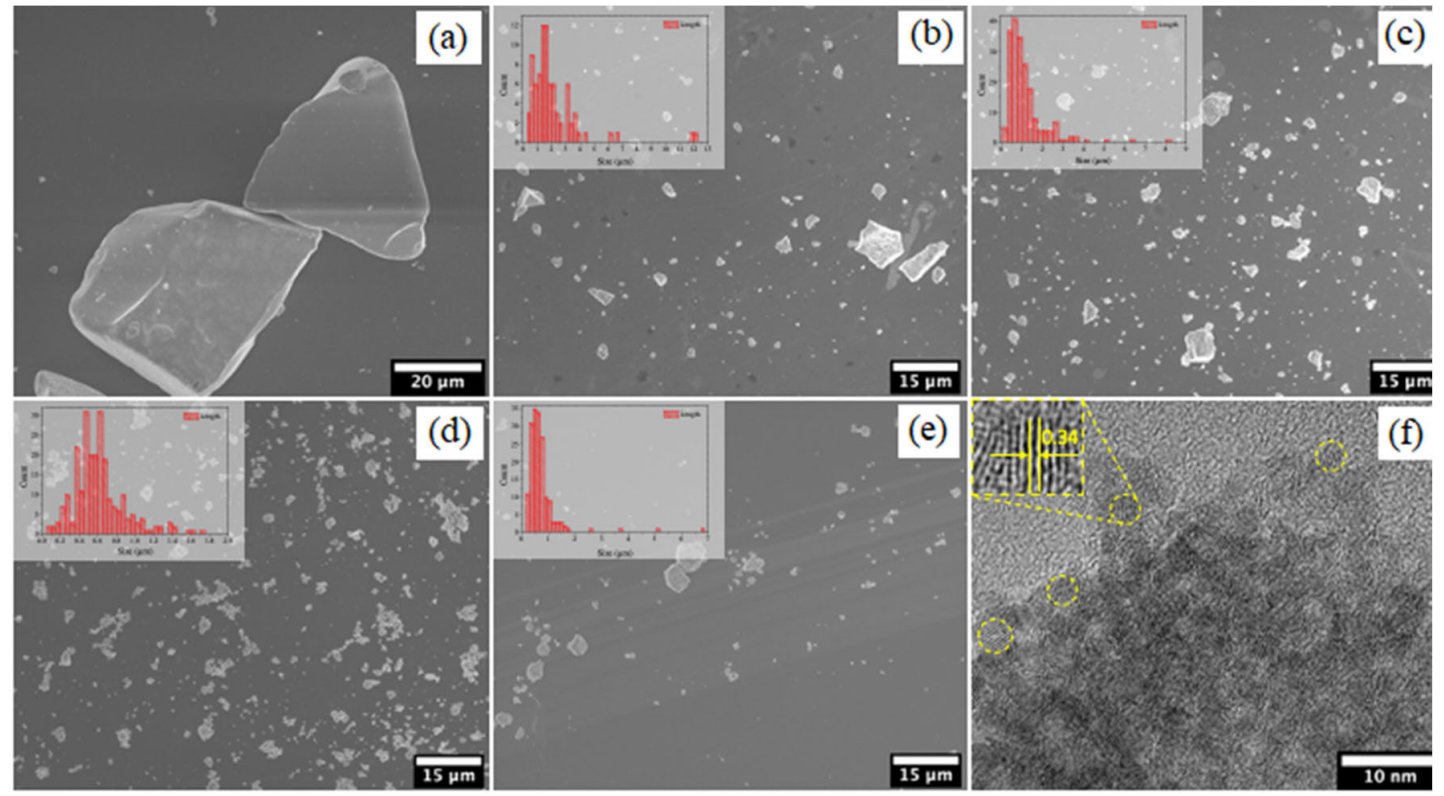

Figure 2. Representative images of the pristine $\mathrm{SnO}_{2}$ sample and processed nanoparticles by milling for different times, from (a) 0 ( $\mathrm{Sn}$-cop), (b) 10 , (c) 30 , (d) 90, (e) $150 \mathrm{~min}$, and (f) HRTEM images of pristine $\mathrm{SnO}_{2}$. 
properties of a semiconductor reveal important parameters of its performance in catalytic processes since the specific surface area can be related to the number of active sites on the material's surface. The sample as prepared $\left(\mathrm{SnO}_{2}\right.$-cop) has a surface area of $188 \mathrm{~m}^{2} \mathrm{~g}^{-1}$, and this value decreases to a minimum of $127 \mathrm{~m}^{2} \mathrm{~g}^{-1}$ in the sample milled for $90 \mathrm{~min}$ (Sn-M90), i.e., 32\% lower. Although the specific surface area of the materials was expected to increase with grinding time, ${ }^{31}$ the result indicates that the compaction of the particles and agglomerates with the collapse of the xerogel structure led to a surface amorphization and decrease in the specific surface area. ${ }^{32}$

The photocatalytic efficiency of the pristine and milling $\mathrm{SnO}_{2}$ samples was monitored by the degradation rate of rhodamine $\mathrm{B}$ dye under UV-light radiation (Figure $3 \mathrm{a}$ ). ${ }^{33}$ Before UV illumination, the catalysts were kept dispersed in the dye solution overnight to achieve adsorption and desorption equilibrium. ${ }^{34}$ No significant discoloration was observed, indicating that this effect may be negligible. However, after illumination, the blank experiments performed without catalyst showed poor (ca. 5\%) selfphotolysis of the dye concentration after 30 min of radiation exposure.

Figure 3a shows the degradation efficiency of all prepared $\mathrm{SnO}_{2}$ nanoparticles. The high efficiencies of Sn-M90 and Sn-M150 demonstrate that the mechanical activation process has a limit of time (90 $\mathrm{min})$, and above that, there is no significant improvement in material performance. The best performance of the milled samples can be attributed to the high-energy ball milling approach. The decrease in secondary particle agglomerates' size promotes particle dispersion in the dye solution, beneficial for photocatalytic performance. ${ }^{35,36}$ Furthermore, the structural distortions make the materials' surface susceptible to coordinate functional groups that boost the photocatalytic efficiency, such as hydroxyl groups. ${ }^{13,37,38}$ It suggests that the effects promoted by grinding alter the photocatalytic performance of $\mathrm{SnO}_{2}$ following the pseudofirst-order kinetic model for $\mathrm{RhB}$ degradation.

Apparent kinetic constants $\left(\mathrm{k}_{\text {app }}\right)$ are in Figure $3 \mathrm{~b}$ and Table 2, showing $\mathrm{R}^{2}$ (coefficient of determination) values higher than 0.95 that confirm rhodamine $B$ photodegradation following the first-order mechanism. The $\mathrm{k}_{\text {app }}$ for the $\mathrm{Sn-M90}$ sample was approximately 10 times higher than the pristine $\mathrm{SnO}_{2}$ and 2 times than the lessgrounded samples, indicating the suitability of the ball mill to mechanically activate metallic oxides by the highly energetic collision between the zircon balls. After 90 min, no gain in $\mathrm{k}_{\mathrm{app}}$ was observed, suggesting that this is an optimum time for the treatment.

Table 2. Apparent reaction rate constants $\left(\mathrm{k}_{\mathrm{app}}\right)$ of the photocatalytic degradation of the $\mathrm{RhB}$ dye, constant of hydroxyl radical formation $\left(\mathrm{k}_{\mathrm{OH}}\right)$

\begin{tabular}{lcccc}
\hline Sample & $\begin{array}{c}\mathrm{k}_{\mathrm{app}} \times 10^{-2} / \\
\mathrm{min}^{-1}\end{array}$ & $\mathrm{R}^{2}$ & $\mathrm{k}_{\mathrm{OH}} / \mathrm{min}^{-1}$ & $\mathrm{R}^{2}$ \\
\hline Sn-cop & 0.479 & 0.961 & 0.30 & 0.961 \\
Sn-M10 & 2.13 & 0.954 & 0.75 & 0.992 \\
Sn-M30 & 2.93 & 0.956 & 1.37 & 0.996 \\
Sn-M90 & 4.90 & 0.974 & 2.02 & 0.993 \\
Sn-M150 & 4.86 & 0.977 & 1.87 & 0.994 \\
\hline
\end{tabular}

$\mathrm{R}^{2}$ : coefficient of determination.

UV-Vis diffuse reflectance was used to measure the optical properties of $\mathrm{SnO}_{2}$ pristine and dry-milled samples. Tauc equation was applied to estimate the bandgap energy of the prepared materials (Figure S1, Supplementary Information (SI) section). ${ }^{39}$ The experimental results indicated that all $\mathrm{SnO}_{2}$ samples show strong photoabsorption below $400 \mathrm{~nm}$ (i.e., in the UV range), attributed to the charge transfer (CT) from the valence band (mainly formed by $\mathrm{O} 2 \mathrm{p}$ orbitals of the oxide anions) to the conduction band (mainly formed by $\mathrm{Sn} 5 \mathrm{p}$ states of the $\mathrm{Sn}^{4+}$ cations). ${ }^{5,40}$ The $\mathrm{SnO}_{2}$ bandgap absorption edge was blue-shifted to $0.53 \mathrm{eV}$,
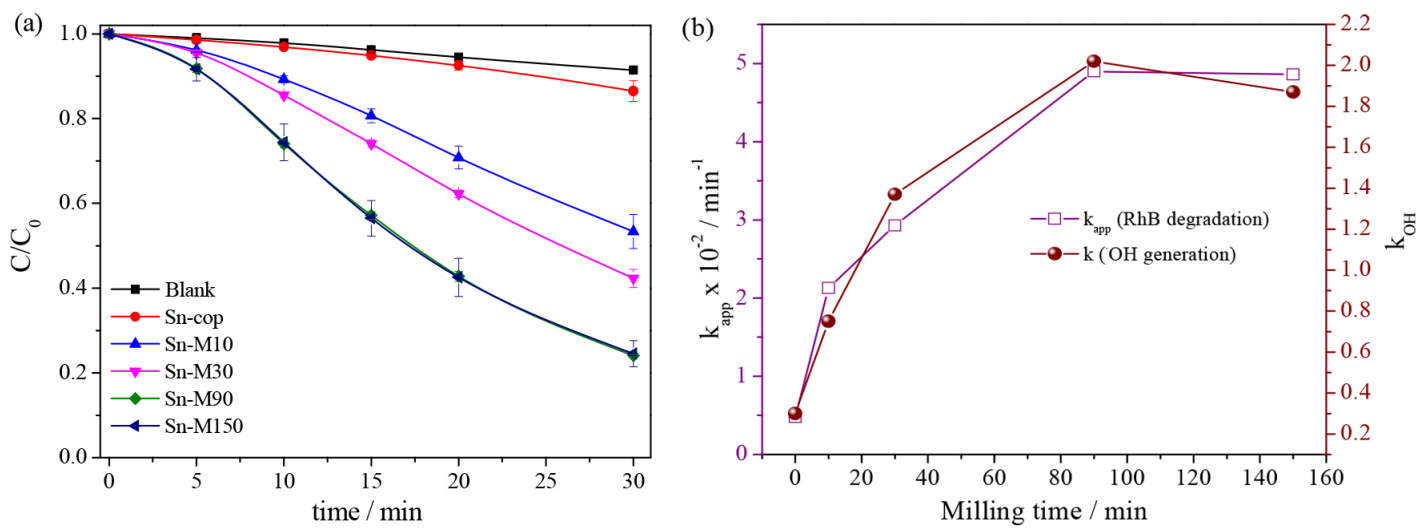

Figure 3. (a) Photocatalytic performance of $\mathrm{SnO}_{2}$ pristine and milled at different times (10, 30, 90, and 150 min) for rhodamine $\mathrm{B}$ ( $\mathrm{RhB}$ ) degradation under UV-light irradiation, and (b) the relation between $\mathrm{k}_{\mathrm{app}}$ and $\mathrm{k}_{\mathrm{OH}}$ for the sample milled for $90 \mathrm{~min}$ (Sn-M90). 
showing that the milling process also promoted changes over the material's optical properties due to the energy involved in the collision, as well as in the crystalline lattice and particle shapes of $\mathrm{SnO}_{2}$, as observed in the XRD results and micrographs, respectively. ${ }^{41,42}$

Widening of the bandgap, in principle, can harm photocatalytic activity by decreasing quantum efficiency. However, the favorable arrangement of these band levels promotes transfers at the interface, initiating various types of thermodynamically inaccessible redox reactions, such as the radical species generation. ${ }^{43}$ Thus, based on the empirical determination of the bandgap values $\left(\mathrm{E}_{\mathrm{g}}\right)$, the positions of the valence $\left(\mathrm{E}_{\mathrm{VB}}\right)$ and conduction band $\left(\mathrm{E}_{\mathrm{CB}}\right)$ of the prepared materials were estimated using the equations 1 and $2: 44$

$\mathrm{E}_{\mathrm{CB}}=\chi-\mathrm{E}_{\mathrm{e}}-0.5 \mathrm{E}_{\mathrm{g}}$

$\mathrm{E}_{\mathrm{VB}}=\mathrm{E}_{\mathrm{CB}}+\mathrm{E}_{\mathrm{g}}$

where $\chi$ is the absolute electronegativity of the semiconductor $\left(\mathrm{SnO}_{2}=6.22 \mathrm{eV}\right)$, and $\mathrm{E}_{\mathrm{e}}$ is the energy of free electrons on the hydrogen scale $(4.5 \mathrm{eV})$. The obtained $\mathrm{E}_{\mathrm{CB}}$ and $\mathrm{E}_{\mathrm{VB}}$ values were illustrated in Figure 4 and compared to aqueous redox potential. The milled materials with enlarged bandgap have more positive VB potential than the pristine, i.e., a stronger oxidation potential. ${ }^{7}$ Furthermore, the conduction band minimum of the $\mathrm{SnO}_{2}$ samples changes from -0.08 to $-0.34 \mathrm{~V}$ after $150 \mathrm{~min}$ of milling, leading to a more favorable reduction of the adsorbed $\mathrm{O}_{2}$ to $\mathrm{O}_{2}^{--}$, and contributing to an improvement in the dye degradation rate. This behavior may be related to the increase in oxidant radical species and the inhibition of photogenerated $\mathrm{e}^{-} / \mathrm{h}^{+}$ pairs recombination. ${ }^{7}$

The surface groups of the synthesized samples have been further examined by DR-NIR (Figure 5). Figure 5a shows the full range spectra of $\mathrm{SnO}_{2}$ pristine and the treated samples at different times. All samples display two main bands with maximum intensity at $6980(1433 \mathrm{~nm})$ and $5170 \mathrm{~cm}^{-1}(1934 \mathrm{~nm})$, as assigned in Table 3. Since the NIR signal depends on the particles' size, the intensities vary among the samples. ${ }^{45}$ For better analysis of the relative composition of each surface group, all the spectra were normalized at $6980 \mathrm{~cm}^{-1}$, i.e., considering equivalent amounts of free water. Thus, it allowed us to estimate, qualitatively, the extension of the presence of $\mathrm{OH}$ groups and adsorbed water on the $\mathrm{SnO}_{2}$ surface by comparing the relative area of the band at around $5170 \mathrm{~cm}^{-1}$. From Figure $5 \mathrm{a}$, it is possible to verify that the band area increases with the milling time, following the trend of photocatalytic experiments, i.e., higher areas are assigned to the samples milled for 90 and 150 min, suggesting that these samples have, proportionally, more $\mathrm{OH}$ groups and adsorbed water on their surfaces. This result is in agreement with the specific surface area values presented in Table 1, demonstrating that the milling process induces some particle aggregation but makes the particle surfaces susceptible to hydroxylation. ${ }^{46}$

The as-prepared $\mathrm{SnO}_{2}$ samples have $\mathrm{OH}$ groups and adsorbed water on the surface, similar to the $\mathrm{TiO}_{2}$ structure described by Takeuchi et al. ${ }^{47}$ The spectra can be deconvoluted into three components, assigned to the different $\mathrm{H}_{2} \mathrm{O}$ molecules' structures, depending on the number of intermolecular hydrogen bonds $\left(S_{1}, S_{2}\right.$, and $\mathrm{S}_{\mathrm{n}}$ ), as seen in Figures $5 \mathrm{~b}$ and $5 \mathrm{c}$ for the combination $\left(\delta+v_{3}\right)$ band of $\mathrm{H}_{2} \mathrm{O}$ adsorbed on Sn-cop and Sn-M90. The wavenumbers of these components are summarized in Table 4 , and our previous study ${ }^{23}$ illustrated the concrete models of the $\mathrm{H}_{2} \mathrm{O}$ molecules with different numbers of hydrogen bonds.

The deconvoluted spectra for the Sn-cop (Figure 5b) and Sn-M90 (Figure 5c) samples show in more detail that each component's contribution follows the same pattern: $\mathrm{S}_{1}$ structure is the most relevant component, followed by $\mathrm{S}_{2}$ and $\mathrm{S}_{\mathrm{n}}$. This sequence suggests that water molecules

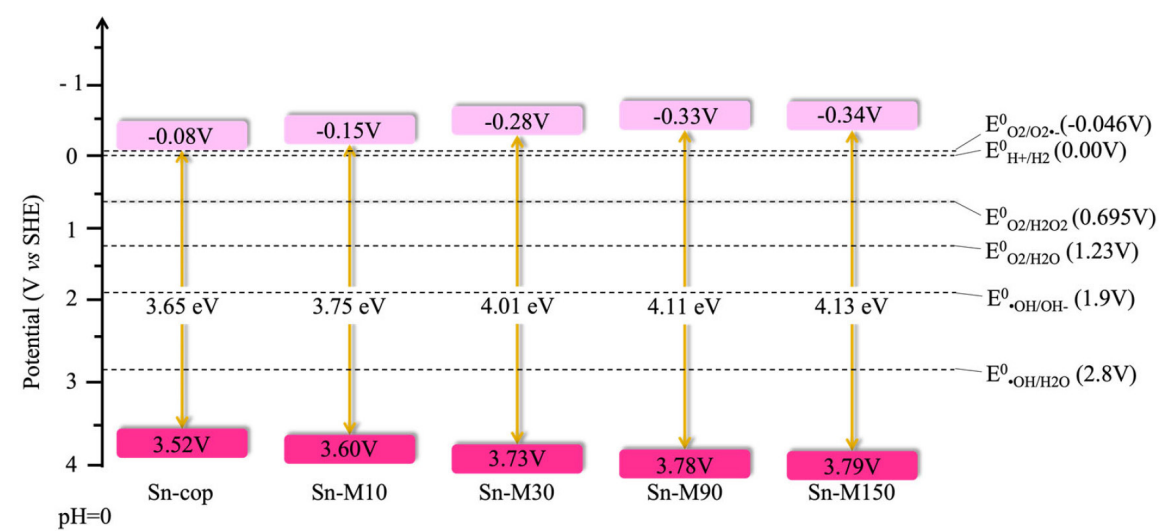

Figure 4. Schematic representation of the valence and conduction band potentials, and bandgap energies of pristine $\mathrm{SnO}_{2}(\mathrm{Sn}$-cop) and samples submitted to different grinding times (Sn-M10, Sn-M30, Sn-M90, and Sn-M150). 


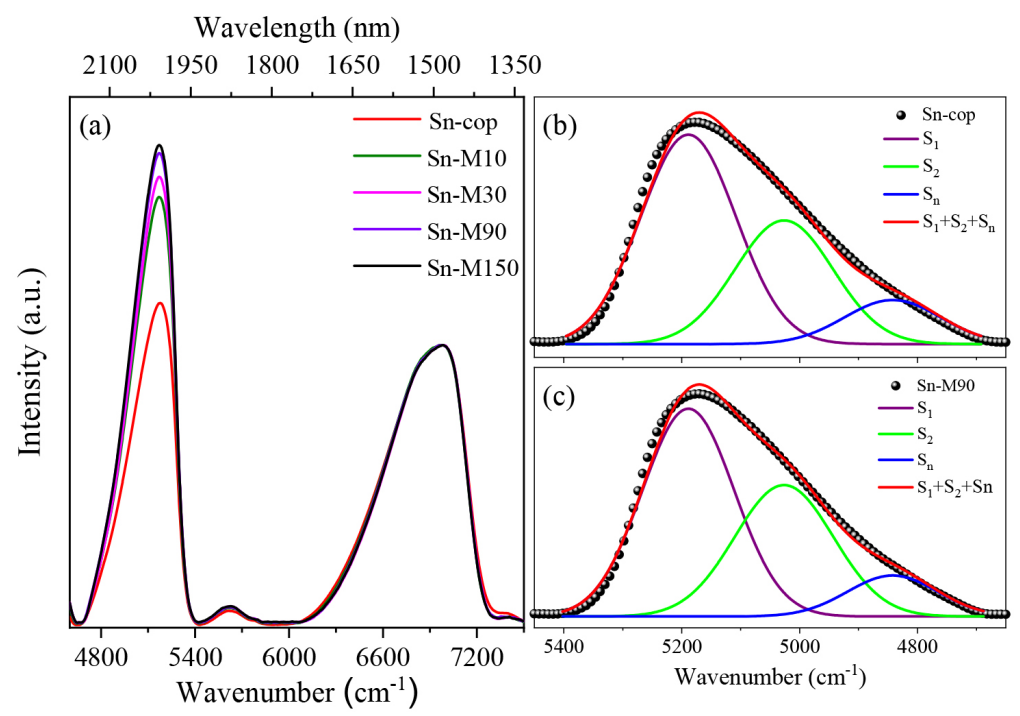

Figure 5. (a) Full NIR spectrum of the untreated and mechanically treated $\mathrm{SnO}_{2}$ samples for different times; deconvolution NIR spectra for (b) Sn-cop and (c) Sn-M90 in the range of 5450 to $4650 \mathrm{~cm}^{-1}$.

Table 3. Assignment of the near-infrared (NIR) bands of the prepared $\mathrm{SnO}_{2}$ samples

\begin{tabular}{lcc}
\hline Band & Vibration mode & Band description \\
\hline $\begin{array}{l}6980 \mathrm{~cm}^{-1} \\
(1433 \mathrm{~nm})\end{array}$ & $\left(v_{1}+v_{3}\right) \mathrm{H}_{2} \mathrm{O}$ & $\begin{array}{c}\text { symmetric }\left(v_{1}\right) \text { and asymmetric }\left(v_{3}\right) \\
\text { stretching vibration modes of } \mathrm{H}_{2} \mathrm{O} \\
\text { molecules }\end{array}$ \\
\hline $\begin{array}{l}5170 \mathrm{~cm}^{-1} \\
(1934 \mathrm{~nm})\end{array}$ & $\left(\delta+v_{3}\right) \mathrm{H}_{2} \mathrm{O}$ & $\begin{array}{c}\text { bending }(\delta) \text { and asymmetric stretching } \\
\left(v_{3}\right) \text { of the physisorbed } \mathrm{H}_{2} \mathrm{O} \text { molecules }\end{array}$ \\
\hline
\end{tabular}

adsorbed on the $\mathrm{SnO}_{2}$ surface have, predominantly, one active hydrogen bond. ${ }^{47}$ Also, compared to the reference values for the three components in the NIR spectrum of liquid-phase $\mathrm{H}_{2} \mathrm{O}$, it shifts toward lower wavenumber regions for the absorbed $\mathrm{H}_{2} \mathrm{O}$ molecules, indicating that the chemical states for the $\mathrm{H}_{2} \mathrm{O}$ molecules are stabilized adsorption process. From the contribution of each component in the spectra, we suggest the formation of $\mathrm{H}_{2} \mathrm{O}$ multilayers, ascribed to the direct interaction of $\mathrm{H}_{2} \mathrm{O}$ molecules with the surface cations or surface hydroxyls on the $\mathrm{SnO}_{2}$ surface. They would form a chemisorbed $\mathrm{H}_{2} \mathrm{O}$ monolayer, being hydrogen-bonded $\mathrm{H}_{2} \mathrm{O}$ molecules form multilayers as physisorbed $\mathrm{H}_{2} \mathrm{O}$ molecules $\left(\mathrm{S}_{1}, \mathrm{~S}_{2}\right.$, and $\left.\mathrm{S}_{\mathrm{n}}\right){ }^{23}$

When the semiconductor surface is rich in hydroxyl groups, as the samples prepared through the milling process, there is a significant improvement in photocatalytic activity due to: $(i)$ an improved semiconductor/dye interaction since they act as Bronsted acids by changing the surface charge of the particles; (ii) formation of trapped holes on $\mathrm{SnO}_{2}$ surface; and (iii) hydroxyl radicals attack. ${ }^{7,13,22}$

Thus, detection of ${ }^{\circ} \mathrm{OH}$ produced during irradiation can provide valuable information about the mechanism of photocatalytic degradation. ${ }^{22,48}$ The rate of hydroxyl radical production $\left(\mathrm{k}_{\mathrm{OH}}\right)$ is estimated by the integrated zero-order rate law (Figure S2, SI section), given in equation 3:

$[\mathrm{A}]=-\mathrm{k}_{\mathrm{OH}} \mathrm{t}+[\mathrm{A}]_{0}$

where [A] is the concentration of 2-hydroxyterephthalic acid, $[\mathrm{A}]_{0}$ is the initial concentration of 2-hydroxyterephthalic acid, $t$ is the reaction time, and $\mathrm{k}_{\mathrm{OH}}$ is the zero-order rate constant of hydroxyl radical formation. A plot of [A] $v s$. $\mathrm{t}$ gives a straight line with a slope of $-\mathrm{k}_{\mathrm{OH}}$, and the values obtained for $\mathrm{k}_{\mathrm{OH}}$ are shown in Table 2 and Figure $3 \mathrm{~b}$ for all samples. In Figure $3 b$, we observe that both $\mathrm{k}_{\mathrm{app}}$ and $\mathrm{k}_{\mathrm{OH}}$ values exhibit the same trend in the function of milling time, confirming that the primary degradation mechanism is the hydroxyl radical attack. Therefore, the mechanical activation of $\mathrm{SnO}_{2}$ favored the collapse of the xerogel

Table 4. Components determined by the deconvolution of combination band $\left(\delta+v_{3}\right)$ at $5170 \mathrm{~cm}^{-1}$

\begin{tabular}{|c|c|c|c|c|c|c|c|c|c|c|}
\hline \multirow{2}{*}{ Sample } & \multicolumn{2}{|c|}{$S_{1\left(5188 \mathrm{~cm}^{-1}\right)}$} & \multicolumn{2}{|c|}{$\mathrm{S}_{2\left(5027 \mathrm{~cm}^{-1}\right)}$} & \multicolumn{2}{|c|}{$\mathrm{S}_{\mathrm{n}\left(4841 \mathrm{~cm}^{-1}\right)}$} & \multicolumn{2}{|c|}{$\mathrm{S}_{1}+\mathrm{S}_{2}+\mathrm{S}_{\mathrm{n}}$} & \multicolumn{2}{|c|}{ Total area } \\
\hline & a. u. & $\%$ & a. u. & $\%$ & a. u. & $\%$ & a. u. & $\%$ & a. u. & $\%$ \\
\hline Sn-cop & 129.2 & 55.6 & 78.1 & 33.6 & 27.4 & 11.8 & 234.7 & 101 & 232.2 & 100 \\
\hline Sn-M90 & 179.4 & 53.9 & 121.7 & 36.6 & 34.7 & 10.4 & 335.8 & 100.9 & 332.9 & 100 \\
\hline
\end{tabular}

$\mathrm{S}_{1}: \mathrm{H}_{2} \mathrm{O}$ with one active H-bond; $\mathrm{S}_{2}: \mathrm{H}_{2} \mathrm{O}$ with active H-bonds; $\mathrm{S}_{\mathrm{n}}: \mathrm{H}_{2} \mathrm{O}$ with two active and one (or two) passive $\mathrm{H}$-bond(s) (polymeric chained $\mathrm{H}_{2} \mathrm{O}$ molecules); a. u.: arbitrary unit. 
network, possibly improving the fragments' dispersion in water. In addition, it would increase the exposure of adsorbed surface groups suitable for radical generation, such as $\mathrm{M}-\mathrm{OH}$ and adsorbed water.

Therefore, the proposed mechanism is based on the improved photocatalysis performance of the prepared materials and the electronic structure (Figure 6). The untreated $\left(\mathrm{SnO}_{2}\right.$-cop) and treated $\left(\mathrm{SnO}_{2}\right.$-M90) samples are activated by the incident UVC radiation since it has higher energy than its bandgap, generating charge carriers $\left(\mathrm{e}^{-} / \mathrm{h}^{+}\right)$. However, the photocatalysis results show that the sample milled for a longer time improves photocatalytic performance. This may be associated with the band position and energy levels generated by the induced mechanical deformations. From the experimental DRS data and theoretical calculations, it could be observed that the position of the valence band of $\mathrm{SnO}_{2}$-cop favors direct oxidation processes $(3.52 \mathrm{~V})$, by the action of the hole $\left(\mathrm{h}^{+}\right)$, or by the formation of hydroxyl radicals $(\cdot \mathrm{OH})$, species with high oxidizing power. ${ }^{49-52}$ However, the conduction band is low energy $(-0.08 \mathrm{~V})$, making complex reactions such as the formation of superoxide radicals $\left(\mathrm{O}_{2}{ }^{-}\right)$, which could act in the oxidation process and increase the lifetime of photogenerated free charge carriers. ${ }^{53,54}$

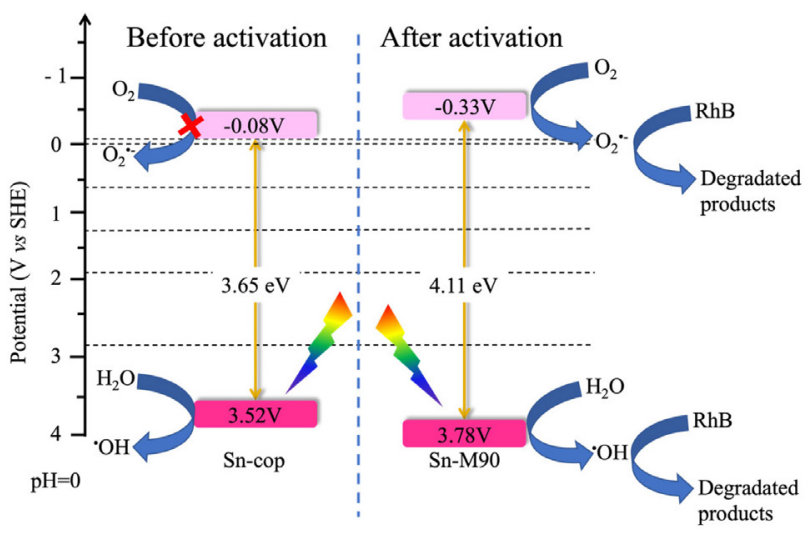

Figure 6. Schematic illustration of the proposed mechanism of activated and pristine $\mathrm{SnO}_{2}$ used in photocatalytic reactions for rhodamine $\mathrm{B}$ degradation.

On the other hand, the grinding process for $90 \mathrm{~min}$ induced a shift of the energy level of the valence band of the $\mathrm{SnO}_{2}$-M90 sample to $3.78 \mathrm{~V}$, i.e., changing the energy in the valence band to more oxidative potentials and contributing to the direct and indirect degradation of the rhodamine $\mathrm{B}$ molecule. This sample also shows a shift of the energy level of the conduction band to more reducing potentials. Thus, the materials prepared during $90 \mathrm{~min}$ of milling can conduct oxidation and reduction reactions efficiently because they generate oxidizing species in the valence band, ${ }^{55}$ and the conduction band, the electron is transferred to the oxygen dissolved in the reaction medium, forming the superoxide radical $\left(\mathrm{O}_{2}^{--}\right)$, which besides contributing to the oxidation process, also increases the lifetime of charge carriers, ${ }^{53,56}$ an essential factor for the photocatalysis process, since the main mechanism for the oxidation process analyzed is driven by hydroxyl radicals $\left({ }^{\circ} \mathrm{OH}\right)$ as shown in Figure 6. ${ }^{22,56}$

In practical terms, catalysis is a surface phenomenon, and since mechanical activation can generate surface-active sites, it directly influences the photocatalytic behavior of materials. Milling breaks the bonds that hold the particles together in the xerogel structure of $\mathrm{SnO}_{2}$, generating point or surface defects. The generation of these defects in ionic solids results in the concentration of ions on the surface and, consequently, in the adsorption of species to balance the system. Therefore, to verify the extent of this effect, the stability of Sn-M90 was evaluated for three consecutive cycles (Figure 7), showing an almost constant behavior, i.e., the photocatalytic activity is kept unaltered along that time. It strongly indicates that the surface modification is permanent, i.e., the interaction points are structural and not due to transient effects.

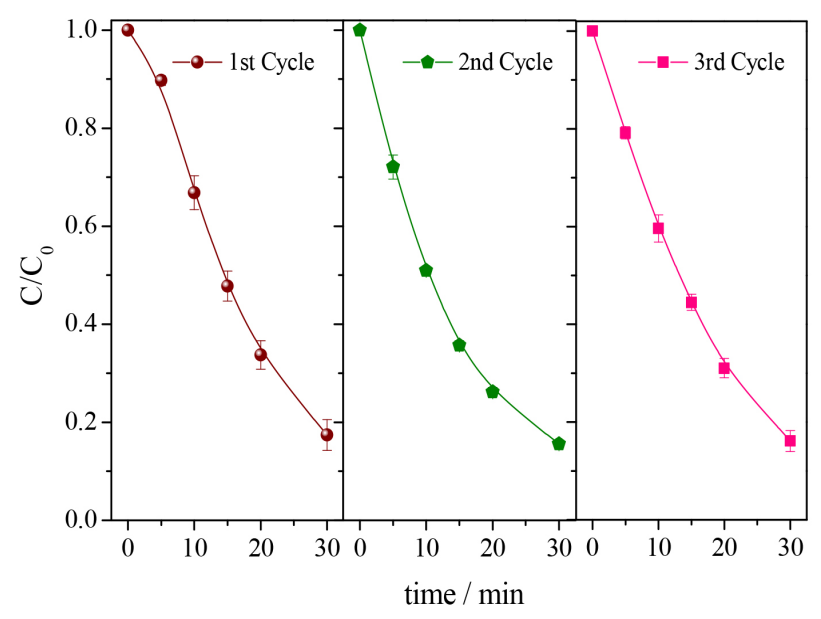

Figure 7. Photocatalytic stability testing of Sn-M90 sample for degradation of rhodamine $\mathrm{B}$ under UV radiation.

\section{Conclusions}

In summary, $\mathrm{SnO}_{2}$ nanoparticles were prepared by precipitation method under mild conditions and activated by solvent-free ball milling at different times. The mechanical friction promoted by the high collision energy induced changes in the crystalline and electronic structure of $\mathrm{SnO}_{2}$, also the breakup of the micrometric particles. Although these slight changes, it was enough to improve the photocatalytic activity of the $\mathrm{SnO}_{2}$ nanoparticles for degradation of rhodamine $\mathrm{B}$ dye under UV radiation. Thus, it was found that the higher milling time was better for photocatalytic application due to modifications on the 
energy level of the valence $\left(\mathrm{E}_{\mathrm{VB}}\right)$ and conduction $\left(\mathrm{E}_{\mathrm{CB}}\right)$ bands and the formation of hydroxyl groups on the surface of the materials. Furthermore, it leads to a decrease in the recombination rate of charge carriers and consequently to an increase in the oxidizing power of the milled $\mathrm{SnO}_{2}$ due to the longer lifetime of electrons and holes pair as well as the generation of superoxide and hydroxyl radicals, species with higher oxidation potential, which have excellent stability, as the material recoveries showed that the activity remained practically unchanged.

\section{Supplementary Information}

Supplementary data (Tauc plot and photoluminescence spectra) are available free of charge at http://jbcs.sbq.org. br as PDF file.

\section{Acknowledgments}

The authors would like to thank the National Council for Scientific and Technological Development (CNPq, grants Nos. 316538/2020-5, 381187/2021-7), the Coordination for the Improvement of Higher Education Personnel (CAPES, finance code 001), the São Paulo Research Foundation (FAPESP, grant No. 2018/01258-5), and the Research Network of Nanotechnology Applied to Agribusiness (AgroNano Network/Embrapa, MCTI-SisNano) for financial support. The authors also thank the Structural Characterization Laboratory (LCE) for the technical support in transmission electron microscopy. Caue Ribeiro also acknowledges Alexander von Humboldt Foundation by Experienced Research Fellowship (CAPES/Humboldt Agreement, Process 88881.145566/2017-1) and Return Grant.

\section{References}

1. Naseem, T.; Durrani, T.; J. Environ. Chem. Ecotoxicol. 2021, 3,59 .

2. Silva, G.; Lopes, O.; Dias, E.; Torres, J.; Nogueira, A.; Faustino, L.; Prado, F.; Patrocínio, A.; Ribeiro, C.; Quim. Nova 2021, 44, 963.

3. Bakbolat, B.; Daulbayev, C.; Sultanov, F.; Beissenov, R.; Umirzakov, A.; Mereke, A.; Bekbaev, A.; Chuprakov, I.; Nanomaterials 2020, 10, 1790.

4. Guo, Q.; Zhou, C.; Ma, Z.; Yang, X.; Adv. Mater. 2019, 31, 1901997.

5. Porte, Y.; Maller, R.; Faber, H.; AlShareef, H. N.; Anthopoulos, T. D.; McLachlan, M. A.; J. Mater. Chem. C 2016, 4, 758.

6. Kim, S. P.; Choi, M. Y.; Choi, H. C.; Mater. Res. Bull. 2016, 74,85 .
7. Nosaka, Y.; Nosaka, A. Y.; Chem. Rev. 2017, 117, 11302.

8. Li, X.; Xie, J.; Jiang, C.; Yu, J.; Zhang, P.; Front. Environ. Sci. Eng. 2018, 12, 14.

9. Chung, H. Y.; Wu, X.; Amal, R.; Ng, Y. H.; Mol. Catal. 2020, 487, 110887.

10. Şahin, E. O.; Dai, Y.; Chan, C. K.; Tüysüz, H.; Schmidt, W.; Lim, J.; Zhang, S.; Scheu, C.; Weidenthaler, C.; Chem. - Eur. J. 2021, 27, 11600.

11. Nakibli, Y.; Mazal, Y.; Dubi, Y.; Wächtler, M.; Amirav, L.; Nano Lett. 2018, 18, 357.

12. Kim, M. G.; Kang, J. M.; Lee, J. E.; Kim, K. S.; Kim, K. H.; Cho, M.; Lee, S. G.; ACS Omega 2021, 6, 10668.

13. de Almeida, J. C.; Corrêa, M. T.; Koga, R. H.; Del Duque, D. M. S.; Lopes, O. F.; da Silva, G. T. S. T.; Ribeiro, C.; de Mendonça, V. R.; New J. Chem. 2020, 44, 18216.

14. Xu, C.; De, S.; Balu, A. M.; Ojeda, M.; Luque, R.; Chem. Commun. 2015, 51, 6698.

15. Szczęśniak, B.; Choma, J.; Jaroniec, M.; Mater. Adv. 2021, 2 , 2510.

16. Santos, D. F. M.; Soares, O. S. G. P.; Figueiredo, J. L.; Pereira, M. F. R.; Environ. Technol. 2020, 41, 117.

17. Šepelák, V.; Bégin-Colin, S.; Le Caër, G.; Dalton Trans. 2012, $41,11927$.

18. Kumar, V.; Choudhary, S.; Malik, V.; Nagarajan, R.; Kandasami, A.; Subramanian, A.; Phys. Status Solidi A 2019, 216, 1900294.

19. Jia, Z.; La, L. B. T.; Zhang, W. C.; Liang, S. X.; Jiang, B.; Xie, S. K.; Habibi, D.; Zhang, L. C.; J. Mater. Sci. Technol. 2017, 33, 856 .

20. Molchanov, V. V.; Buyanov, R. A.; Tsybulya, S. V.; Kryukova, G. N.; Shmakov, A. N.; Boronin, A. I.; Volodin, A. M.; Kinet. Catal. 2004, 45, 684.

21. Ribeiro, C.; Lee, E. J. H.; Giraldi, T. R.; Longo, E.; Varela, J. A.; Leite, E. R.; J. Phys. Chem. B 2004, 108, 15612.

22. da Silva, G. T. S. T.; Carvalho, K. T. G.; Lopes, O. F.; Gomes, E. S.; Malagutti, A. R.; Mastelaro, V. R.; Ribeiro, C.; Mourão, H. A. J. L.; ChemCatChem 2017, 9, 3795.

23. Torres, J. A.; da Silva, G. T. S. T.; Silva, F. B. F.; Ribeiro, C.; ChemPhysChem 2020, 21, 2392.

24. Arbain, R.; Othman, M.; Palaniandy, S.; Miner. Eng. 2011, $24,1$.

25. Carvalho, M. H.; Piton, M. R.; Lemine, O. M.; Bououdina, M.; Galeti, H. V. A.; Souto, S.; Pereira, E. C.; Gobato, Y. G.; de Oliveira, A. J. A.; Mater. Res. Express 2018, 6, 025017.

26. Qadri, S. B.; Skelton, E. F.; Hsu, D.; Dinsmore, A. D.; Yang, J.; Gray, H. F.; Ratna, B. R.; Phys. Rev. B 1999, 60, 9191.

27. Sim, K.-H.; Wang, G.; Kim, T.-J.; Ju, K.-S.; J. Alloys Compd. 2018, 741, 1112 .

28. Bodaghi, M.; Mirhabibi, A. R.; Zolfonun, H.; Tahriri, M.; Karimi, M.; Phase Transitions 2008, 81, 571.

29. Ellouzi, I.; Oualid, H. A.; Proceedings 2018, 3, 3.

30. Anand, K.; Varghese, S.; Kurian, T.; Powder Technol. 2015 , 271,187 
31. Hidalgo, M. C.; Colón, G.; Navío, J. A.; J. Photochem. Photobiol., A 2002, 148, 341.

32. Bégin-Colin, S.; Gadalla, A.; Le Caër, G.; Humbert, O.; Thomas, F.; Barres, O.; Villiéras, F.; Toma, L. F.; Bertrand, G.; Zahraa, O.; Gallart, M.; Hönerlage, B.; Gilliot, P.; J. Phys. Chem. C 2009, 113, 16589.

33. Li, Y.; Yang, Q.; Wang, Z.; Wang, G.; Zhang, B.; Zhang, Q.; Yang, D.; Inorg. Chem. Front. 2018, 5, 3005.

34. Soares, E. T.; Lansarin, M. A.; Moro, C. C.; Braz. J. Chem. Eng. 2007, 24, 29.

35. Kunz, L. Y.; Hong, J.; Riscoe, A. R.; Majumdar, A.; Cargnello, M.; J. Photochem. Photobiol. 2020, 2, 100004.

36. Pavlović, V. P.; Vujančević, J. D.; Mašković, P.; Ćirković, J.; Papan, J. M.; Kosanović, D.; Dramićanin, M. D.; Petrović, P. B.; Vlahović, B.; Pavlović, V. B.; J. Am. Ceram. Soc. 2019, 102, 7735.

37. Nogueira, A. E.; Ribeiro, L. S.; Gorup, L. F.; Silva, G. T. S. T.; Silva, F. F. B.; Ribeiro, C.; Camargo, E. R.; Int. J. Photoenergy 2018, 2018, ID: 6098302.

38. da Silva, G. T. S. T.; Nogueira, A. E.; Oliveira, J. A.; Torres, J. A.; Lopes, O. F.; Ribeiro, C.; Appl. Catal., B 2019, 242, 349.

39. Avis, C.; Jang, J.; Crystals 2021, 11, 851.

40. Batzill, M.; Diebold, U.; Prog. Surf. Sci. 2005, 79, 47.

41. Pang, G.; Chen, S.; Koltypin, Y.; Zaban, A.; Feng, S.; Gedanken, A.; Nano Lett. 2001, 1, 723.

42. Sharma, V.; J. Sol-Gel Sci. Technol. 2017, 84, 231.

43. Yan, H.; Wang, X.; Yao, M.; Yao, X.; Prog. Nat. Sci.: Mater. Int. 2013, 23, 402.

44. Cadan, F. M.; Ribeiro, C.; Azevedo, E. B.; Appl. Surf. Sci. 2021, 537, 147904.
45. Lai, X.; Zheng, Y.; Ipsen, H.; Jacobsen, S.; Larsen, J. N.; Løwenstein, H.; Søndergaard, I.; Appl. Spectrosc. 2007, 61, 1184.

46. Liu, H.; Chen, T.; Chang, J.; Zou, X.; Frost, R. L.; J. Colloid Interface Sci. 2013, 398, 88.

47. Takeuchi, M.; Martra, G.; Coluccia, S.; Anpo, M.; J. Phys. Chem. B 2005, 109, 7387.

48. Ishibashi, K.; Fujishima, A.; Watanabe, T.; Hashimoto, K.; Electrochem. Commun. 2000, 2, 207.

49. Fang, M.-M.; Shao, J.-X.; Huang, X.-G.; Wang, J.-Y.; Chen, W.; J. Mater. Sci. Technol. 2020, 56, 133.

50. Liu, J.; Hodes, G.; Yan, J.; Liu, S. (F.); Chin. J. Catal. 2021, 42, 205

51. Bai, J.; Shen, R.; Chen, W.; Xie, J.; Zhang, P.; Jiang, Z.; Li, X.; Chem. Eng. J. 2022, 429, 132587.

52. Shen, R.; He, K.; Zhang, A.; Li, N.; Ng, Y. H.; Zhang, P.; Hu, J.; Li, X.; Appl. Catal., B 2021, 291, 120104.

53. Hayyan, M.; Hashim, M. A.; AlNashef, I. M.; Chem. Rev. 2016, 116, 3029.

54. Nosaka, Y.; Nakamura, M.; Hirakawa, T.; Phys. Chem. Chem. Phys. 2002, 4, 1088.

55. Xu, X.; Sun, Y.; Fan, Z.; Zhao, D.; Xiong, S.; Zhang, B.; Zhou, S.; Liu, G.; Front. Chem. 2018, 6, 64.

56. Parrino, F.; Livraghi, S.; Giamello, E.; Ceccato, R.; Palmisano, L.; ACS Catal. 2020, 10, 7922.

September 28, 2021

Published online: January 12, 2022 